\title{
Transition process of abrupt climate change based on global sea surface temperature over the past century
}

\author{
Pengcheng Yan ${ }^{1}$, Wei Hou ${ }^{2}$, and Guolin Feng ${ }^{2}$ \\ ${ }^{1}$ Institute of Arid Meteorology, China Meteorological Administration, Lanzhou 730000, China \\ ${ }^{2}$ National Climate Center, China Meteorological Administration, Beijing 100081, China \\ Correspondence to: Guolin Feng (fenggl@cma.gov.cn)
}

Received: 13 January 2016 - Published in Nonlin. Processes Geophys. Discuss.: 5 February 2016

Revised: 11 April 2016 - Accepted: 28 April 2016 - Published: 19 May 2016

\begin{abstract}
A new detection method has been proposed to study the transition process of abrupt climate change. With this method, the climate system transiting from one stable state to another can be verified clearly. By applying this method to the global sea surface temperature over the past century, several climate changes and their processes are detected, including the start state (moment), persist time, and end state (moment). According to the spatial distribution, the locations of climate changes mainly have occurred in the Indian Ocean and western Pacific before the middle twentieth century, in the 1970s in the equatorial middle-eastern Pacific, and in the middle and southern Pacific since the end of the twentieth century. In addition, the quantitative relationship between the transition process parameters is verified in theory and practice: (1) the relationship between the rate and stability parameters is linear, and (2) the relationship between the rate and change amplitude parameters is quadratic.
\end{abstract}

\section{Introduction}

Including a variety of factors, the climate system is a gigantic and complex system. Each member of the system follows a certain rule, and there is a certain interaction between members. According to previous work (Goldblatt et al., 2006; Alexander et al., 2012; Baker and Charlson, 1990; Charney and DeVore, 1979; Zerkle et al., 2012), the climate system has two or more stable states, and the system transiting from one state to another is called climate change (Thom, 1972; Lorenz, 1963, 1976; Rial, 2004). Climate change has an important impact on social politics and economic environments, and it is closely related to human survival, production, and daily life. The climate change issue has aroused great concern in the international community (IPCC, 2014). When climate changes, the system jumps from one state to another, and it experiences a process (Li et al., 1996; Yan et al., 2012, 2013). Most traditional theories and detection methods (Wei, 1999; Feng et al., 2011; He et al., 2012) have been focused on the changes in statistics before and after climate change, such as Yamamoto (Yamamoto et al., 1986), Mann-Kendall (Mann, 1945; Kendall, 1955; Kendall et al., 1976), moving $t$ test, moving cut data-approximate entropy (He et al., 2009; Jin et al., 2015), and the duration has been ignored. Therefore, it is urgent to study the transition process of climate change to understand how the system changes abruptly. By understanding the climate change events from the perspective of the transition period, more phenomenon about climate change would be exposed. Thus, a new concept about the transition process of climate change has been proposed, and the detection method was created (Yan et al., 2015). By referring to the work of $\mathrm{Fu}$ and Wang (1992), there are four types of climate change: change in mean, change in variance, change in trend, and change of seesaw. All four kinds of climate change could be transformed to be "change in mean" with mathematical methods; therefore, the detection method focuses mainly on climate change in mean. Five climate change processes of the Pacific decadal oscillation index were identified (Yan et al., 2015) and the climate change process with a $500 \mathrm{hPa}$ temperature field was also analyzed (Yan et al., 2014).

When the climate system transits from one stable state to another via a process, the duration could be identified no matter how long the period lasts. Muldelsee (2000) developed a regression technique to identify such processes with a ramp 
function. In the present paper, a traditional model (logistic model) is used to regress a real-time sequence. The parameters of the model obtained by regressing the time series. The mode could represent different degrees of change. It has been noticed that climate change has a relationship with the length of time sequence (Yan et al., 2015); thus, a sub-sequence is extracted from the entire sequence for regression. A group of parameters can be obtained when the sub-sequence moves on the entire time sequence. By using the percentile threshold method on the parameters with a given threshold (98\%), climate changes are determined. Based on the concept of climate change process, several climate changes in global sea surface temperature (GSST) have been detected in the past century.

\section{Method and data}

Details of the proposed method have been thoroughly discussed (Yan et al., 2015); a brief description is as follows. A biological model was created, and it is a complex system (May, 1976). The model also describes an abrupt change in mean (Liu and Peng, 2004) and it is expressed as

$\dot{x}=\kappa x(x-\mu)$.

By rewriting Eq. (1) in its difference form $x(t+1)=x(t)+$ $\tau \kappa x(t)(x(t)-\mu))$, this equation can be solved. According to the solution, this model describes a system that transitions from one state to another, and the two states are $x=0, x=\mu$. To help the model handle the more general case in which the system transitions from one state $(x=v)$ to another $(x=\mu)$, it can be modified as follows:

$\dot{x}=\kappa(x-\mu)(v-x)$.

The logistic model and its modified form have been used to study abrupt change in many fields (Guttal and Jayaprakash, 2008). The physical meanings of the parameters $(\mu, \nu, \kappa)$ were thoroughly discussed (Yan et al., 2014, 2015). This paper introduces how to estimate the parameters based on a time series.

A piecewise function is created to describe a curve, which is similar to the preceding one and is divided into three stages:

$x= \begin{cases}v & \text { stage 1 } \\ h \cdot t+\xi & \text { stage 2 } \\ \mu & \text { stage 3 }\end{cases}$

In stages 1 and 3, the system stays in two states, $x=v, x=$ $\mu$. The parameters can be expressed as

$\nu=\frac{1}{n_{1}} \sum_{i=1}^{n_{1}} x_{i}, \quad \mu=\frac{1}{n_{3}} \sum_{i=n_{2}+1}^{n_{1}}+n_{2}+n_{3} x_{i}$,

where $n_{1}$ is the persistence time in stage 1 and $n_{3}$ is the persistence time in stage 3 .
In stage 2, the system is in transition from state $v$ to state $\mu$. Assuming that the transition process is linear, the slope of the process is defined as the rate of change. Based on two points of the process, $A\left(t_{a}, x_{a}\right)$ and $B\left(t_{b}, x_{b}\right)$, the parameter $h$ can be expressed as

$h=\frac{x_{b}-x_{a}}{t_{b}-t_{a}}$.

The parameters $\alpha, \beta$ are defined to describe the two points' locations:

$\left\{\begin{array}{l}x_{\alpha}=\alpha(\mu-v)+v \\ x_{\beta}=\beta(\mu-v)+v\end{array}\right.$,

and the solution (as follows) of the model is to describe the points' locations too.

$t=\frac{1}{\kappa(\mu-v)} \ln \left(\frac{x_{0}-\mu}{x_{0}-v} \cdot \frac{x-v}{x-\mu}\right)+t_{0}$

Based on Eqs. (5)-(7), the parameter $h$ can be expressed as

$$
\begin{aligned}
& h=\frac{\alpha(\mu-v)-\beta(\mu-v)}{\frac{1}{\kappa(\mu-v)}\left(\ln \left(\frac{\alpha(\mu-\nu)}{\alpha(\mu-\nu)-\mu+v} \cdot \frac{\beta(\mu-v)-\mu+v}{\beta(\mu-\nu)}\right)\right)} . \\
& =\kappa(\mu-v)^{2} \frac{\alpha-\beta}{\ln \left(\frac{\alpha}{\beta} \cdot \frac{1-\beta}{1-\alpha}\right)}=\kappa \omega^{2} \chi
\end{aligned}
$$

The new location parameter is defined as $\chi=\frac{\alpha-\beta}{\ln \left(\frac{\alpha}{\beta} \cdot \frac{1-\beta}{1-\alpha}\right)}$, and a change amplitude parameter is defined as $\omega=\mu-v$. Yan et al. (2015) discovered that $\chi$ varies only slightly when the values of $\alpha, \beta$ fall within a certain range. And when $\alpha=0.2, \beta=0.8, \chi(=0.2164)$ is constant.

According to Eq. (3), parameter $h$ can be estimated with the least-squares method:

$h=\sum_{i=n_{1}+1}^{n_{1}+n_{2}} \bar{t}_{i} \cdot \bar{x}_{i} / \sum_{i=n_{1}+1}^{n_{1}+n_{2}} \bar{t}_{i}^{2}$,

where $n_{2}$ is the persistence time in stage 2 . Then parameter $\kappa$ can be expressed as

$\kappa=\frac{h}{(\mu-v)^{2} \chi}$.

Based on Eqs. (4) and (9), parameters $\mu, v, h$ can be estimated optimally by changing $n_{1}, n_{2}, n_{3}$, and parameter $\kappa$ can be calculated using Eq. (10).

Note that parameter $\kappa$ is a stability parameter, which means that the larger its value, the more unstable the system becomes. The quantitative relationship among the rate of change $h$, the stability parameter $\kappa$, and the change amplitude $\omega$ is shown in Eq. (8). The relationship between the rate of change $h$ and the stability parameter $\kappa$ is linear, but that between the rate of change $h$ and the change amplitude $\omega$ is parabolic. According to a numerical test, which applied 
this method to several ideal models, these relationships were clearly verified. The test showed that the ratio of the rate of change and the stability parameter is constant when the change amplitude is fixed. Moreover, when the stability parameter is fixed, the ratio of the rate of change and the square of the change amplitude is also constant.

By applying this method to the Pacific decadal oscillation index, Yan et al. (2015) verified its transition process. In the present paper, this method is applied to analyze the GSST transition process. It is noticed that the sub-sequence could be set as 10a, 20a, 30a, and 40a, and for our purposes the sub-sequence was set as 10a.

The data used in the present paper were reconstructed by the National Oceanic and Atmospheric Administration (2016, http://www.esrl.noaa.gov/psd/data/gridded/data.noaa. ersst.html). The time span of the monthly data is from January 1854 to November 2012, and the spatial resolution is $2^{\circ} \times 2^{\circ}$. These data have been determined to be reliable (Hirahara et al., 2014; Liu et al., 2015; Ratna et al., 2015). During the calculation, the time series of each grid was processed for anomalies per month.

\section{Spatial positions of abrupt changes}

\subsection{Abrupt changes at different start/end moments}

The detection method is applied to identify the temperature sequence in each grid. In order to confirm the climate change, all start moments and end moments were counted. As shown in Fig. 1, the frequencies of start moments and end moments are displayed from 1854 to 2010 . In order to confirm the number of abrupt changes, a threshold of the frequencies was set by an ideal numerical experiment. In the experiment, each original sea surface temperature (SST) series is replaced by a new time series, which is rebuilt by a shuffle algorithm (it is an algorithm to disturb a time series randomly). The new random series has the same mean and variance with the original one. By detecting its abrupt changes with the novel method, the frequencies of start moments and end moments are counted as seen in Fig. 1, and all frequencies are less than $1 \%$. Then, when the frequencies of start moments in $1878,1942,1976$, and two other periods (1890-1920, 19902010) are larger than $1 \%$ (Fig. 1a), the abrupt changes are considered as the real abrupt changes. While according to end moments (Fig. 1b), the frequencies are in 1886, 1950, and 1982 and two other periods (1900-1930, 1990-2010). By comparing the two frequencies, a certain corresponding relationship shows that the climate changes starting in periods of Fig. 1a and ending in periods of Fig. 1b as marked by the red arrows and blue boxes, respectively. For the climate change starting in 1878 and ending in 1886, the frequency of the former is larger than the latter. The reason maybe that some grids started to change in 1878 , while some of these grids did not end in 1886 . The same situation occurs in other periods.

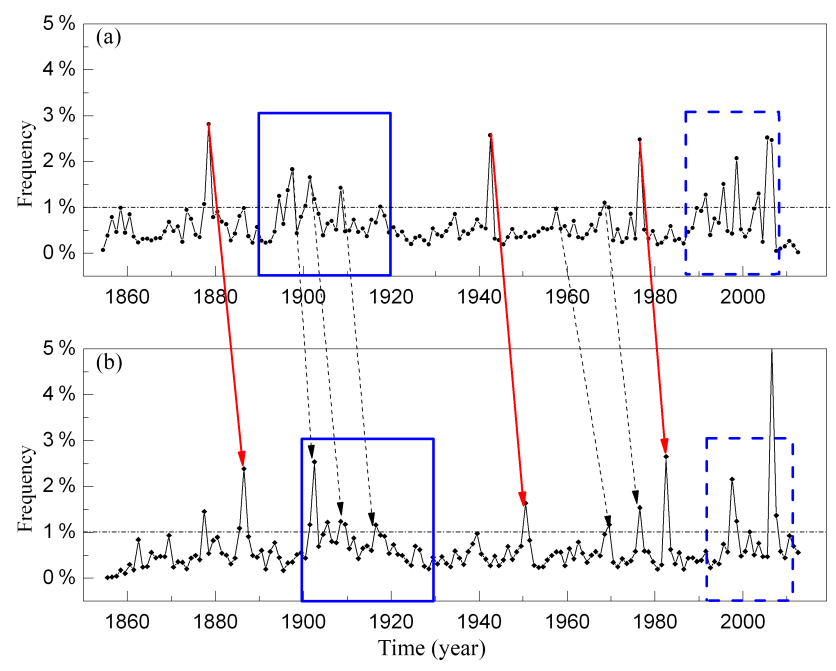

Figure 1. Frequencies of climate changes based on start moments (a) and end moments (b).

In order to verify the corresponding relationship between the frequencies, the spatial distribution of abrupt change, classified based on the start/end moments, is shown in Fig. 2.

1. As shown in Fig. 2a, the abrupt change starting in 1878 occurred mainly in the northern Indian Ocean, part of the central North Pacific and South Pacific, and the equatorial Atlantic region. These regions coincided with those of abrupt change ending in 1886 (Fig. 2b), which indicates that they belonged to the same abrupt change process $(\mathrm{ACP})$.

2. As shown in Fig. 2c, the spatial distribution of abrupt change starting in 1942 coincided with that ending in 1950 (Fig. 2d), mainly covering the coastal regions in the northern and western Indian Ocean and part of the equatorial Atlantic regions. This indicates that they are part of the same ACP.

3. As shown in Fig. 2e, the abrupt change starting in 1976 mainly occurred in the middle-eastern equatorial Pacific Ocean and small regions of the South Pacific near the South Pole. These regions completely coincided with those of the abrupt change ending in 1982 (Fig. 2f), indicating that they were part of the same ACP.

4. As shown in Fig. 2g, the abrupt change starting in 1890 1920 may be divided into three principle periods: that in 1890-1898 occurred mainly in the Indian and South Pacific oceans; that in 1900-1993 occurred mainly in the North Pacific and Atlantic; and the largest one in 1908-1909 occurred mainly in the eastern and western regions of the equatorial Pacific. This coincides with the spatial distributions (Fig. 2h) of abrupt change ending in 1902-1903, 1896-1898, and 1908-1910, and proves that they are part of the same ACP. 

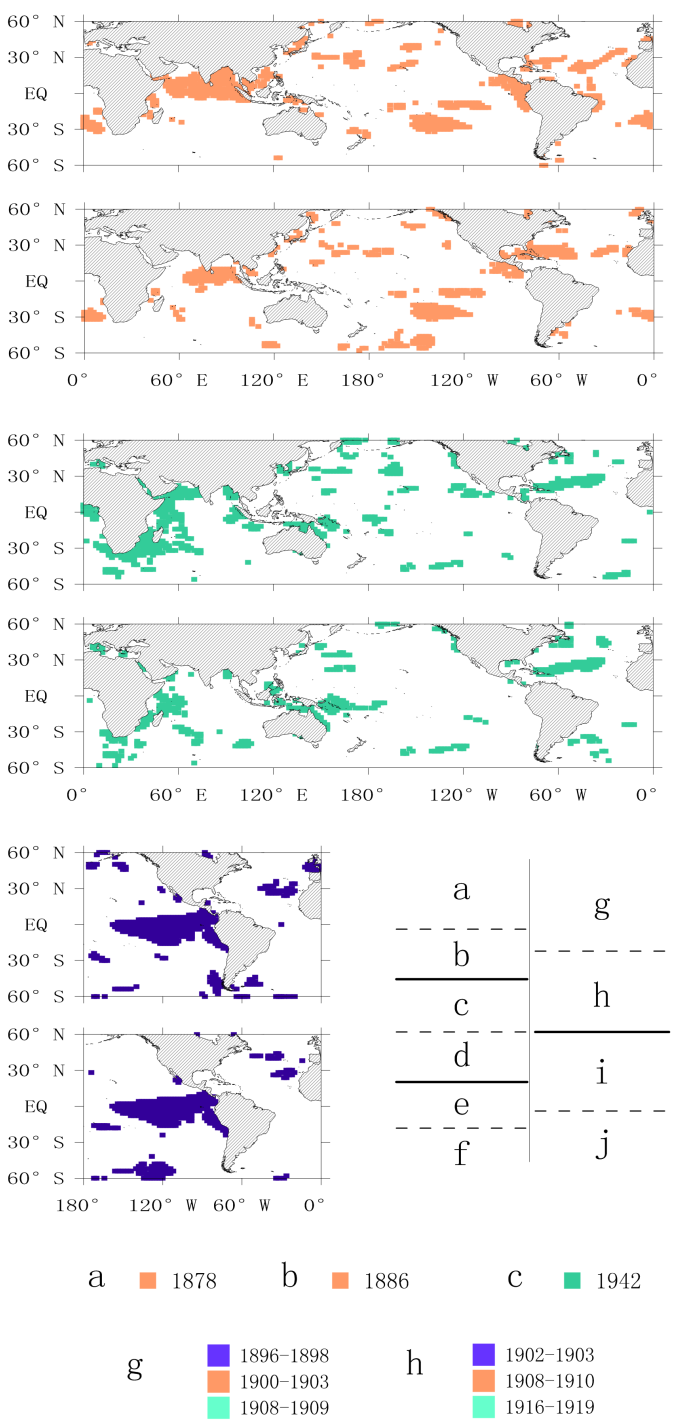
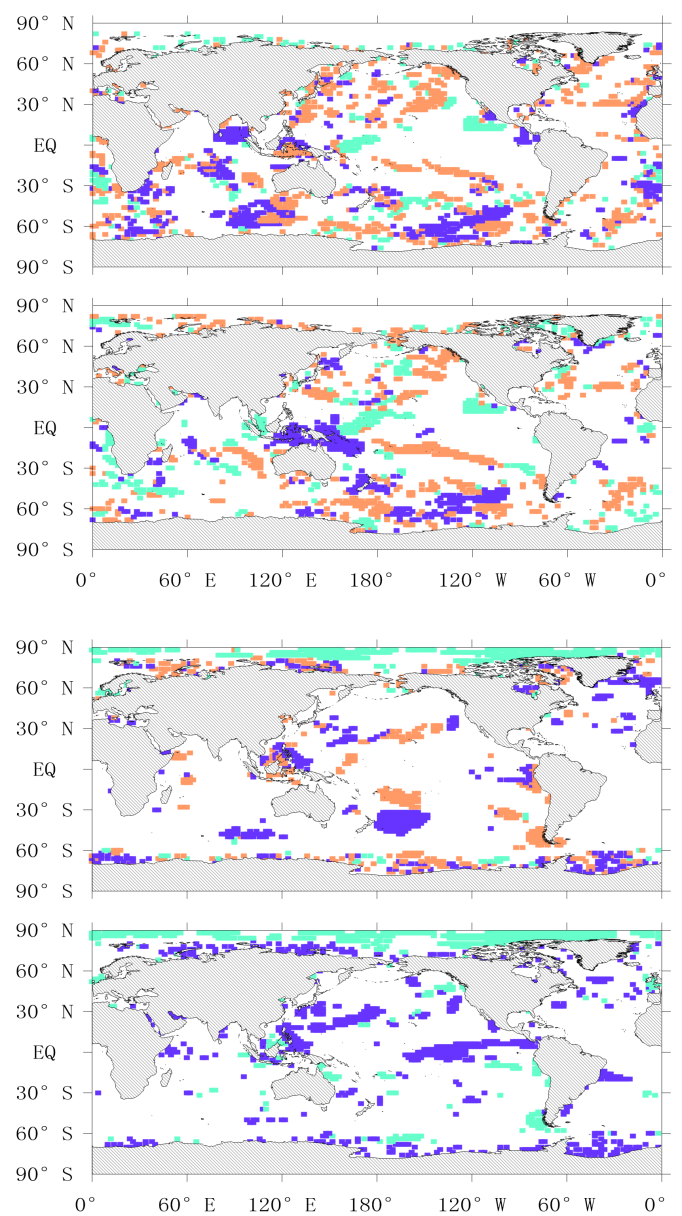

d 1950

e

f

1982
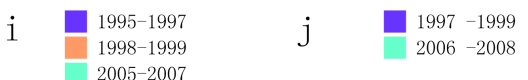

Figure 2. Spatial distribution classified based on frequency of start and end moments of abrupt change. (a) and (b) are the climate changes starting in 1878 and ending in 1886. (c) and (d) are the climate changes starting in 1942 and ending in 1950. (e) and (f) are the climate changes starting in 1976 and ending in 1982. (g) and (h) are the climate changes starting in 1890-1920 and ending in 1990-2010. (i) and (j) are the climate changes starting in 1990-2010 and ending in 1990-2010.

5. As shown in Fig. 2i, the abrupt change, which occurred in 1990-2010, may be divided into three periods: that in 1995-1997 occurred mainly in the western region of the South Pacific; that in 1998-1999 also occurred mainly in the western South Pacific and some regions of the North Pacific; and the most recent one occurred in 2005 and 2007, mainly in the Arctic region. In addition, the abrupt changes starting in 1995-1997 ended in 19971999; and that starting in 1998-1999 and 2005-2007 in the Arctic region ended in 2006-2008 (Fig. 2j). These indicate that they are part of the same abrupt change.

The above analysis verifies that the abrupt change of a certain grid point undergoes three steps: start, sustaining, and end. The spatial distribution situation of the entire ACP co- incides to a large degree with the distribution situation of the abrupt change "point" proposed by Xiao and Li (2007). This indicates that abrupt changes detected by the traditional ACP are contained within the ACP.

It is obvious that the abrupt changes starting in 1878 and 1942 mainly occurred in the Indian Ocean, and the Indian Ocean dipole (IOD) for these two years got strong negative phase and strong positive phase respectively (Suryachandra et al., 2002), which could be the trigger of the abrupt changes. The abrupt change starting in 1976 is known as a climate shift, and most climate elements were detected to experience abrupt change. The abrupt changes of 1890-1920 occurred mainly in the Pacific Ocean, and it is associated with the Interdecadal Pacific oscillation (IPO) index, which 

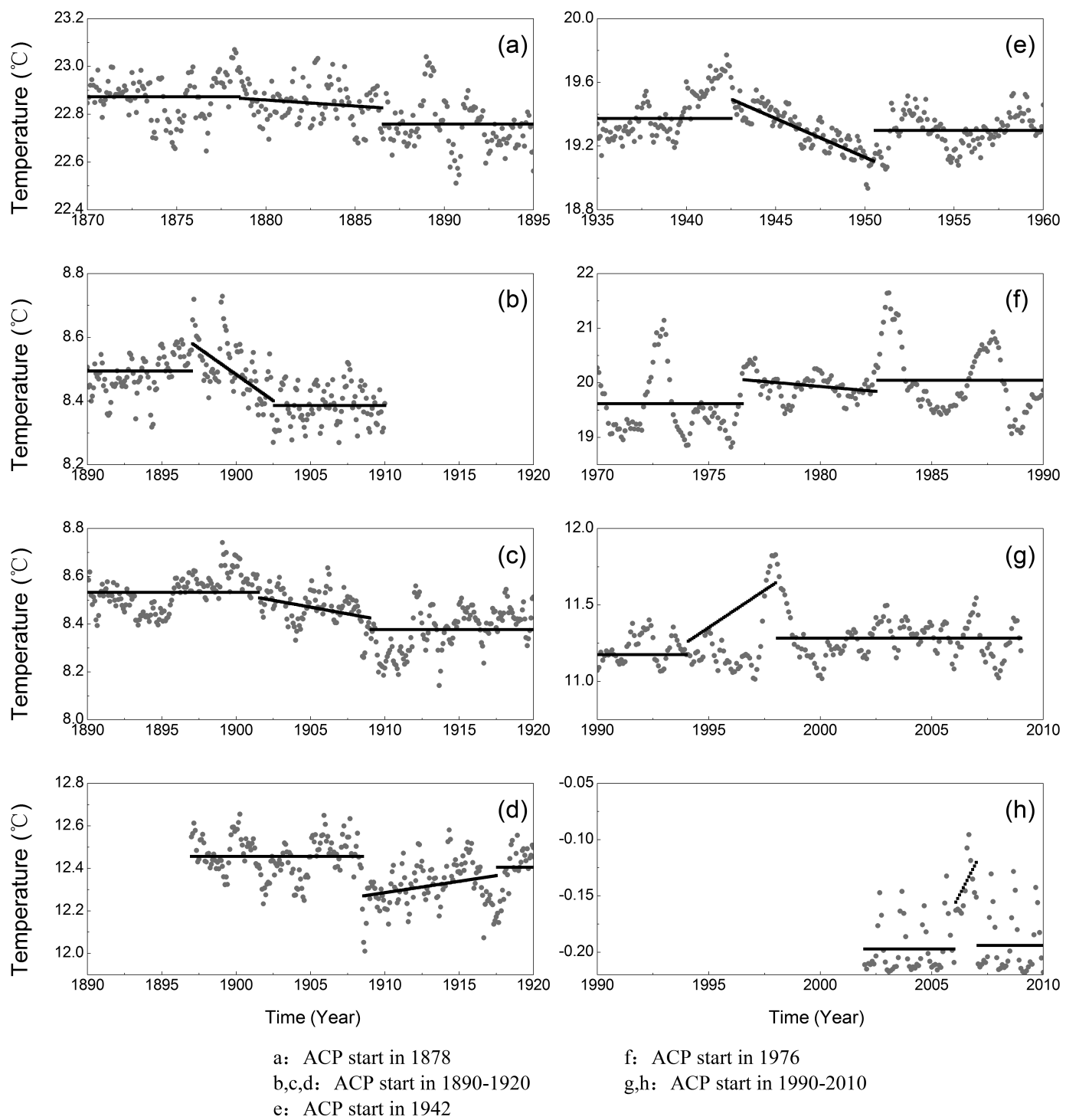

Figure 3. Time sequence average of abrupt changes occurring under the background of the same abrupt change: (a) is the abrupt change starting in 1878; (b-d) is the abrupt change occurring in 1890-1920, including the following: (e) is the abrupt change starting in 1942, (f) is the abrupt change starting in 1976, and (g-h) is the abrupt change starting in 1990-2010.

transits from a negative phase to a positive phase during this period. The abrupt changes starting at the end of 2000 s mainly occurred in high latitudes, which leads to a significant increase of temperature in the polar.

\subsection{Time sequence variation of abrupt change}

By studying the abrupt changes, which occurred in 1878 , 1942, and 1976 and two other periods (1890-1920, 19902010), the averages of the spatial grid points of climate change are calculated. Seven sequences are obtained to describe the transition process as shown in Fig. 3.

In terms of sequence variation, the climate changes occurring before 1960 were characterized by low temperature. For that occurring in 1878 , the temperature of the abrupt changes decreased by an average of $0.12^{\circ}$; the temperatures of the three abrupt changes in $1890-1920$ decreased by $0.11,0.16$, and $0.06^{\circ}$, and that of the abrupt change in 1942 decreased by $0.08^{\circ}$. The abrupt changes occurring after 1960 were characterized by a temperature rise. The temperature of the abrupt change occurring in 1976 increased by $0.35^{\circ}$, and that of the abrupt change in 1990-2010 increased by $0.11^{\circ}$. In addition, for the two abrupt changes occurring at the two other periods (1890-1920 and 1990-2010), due to the fact that the distribution latitude was high, the average temperature of the sequence was about $8-10^{\circ}$ lower than the average temperature of other three abrupt changes occurring at 1878, 1942, and 1976 , which was about $20^{\circ}$. 
(a)

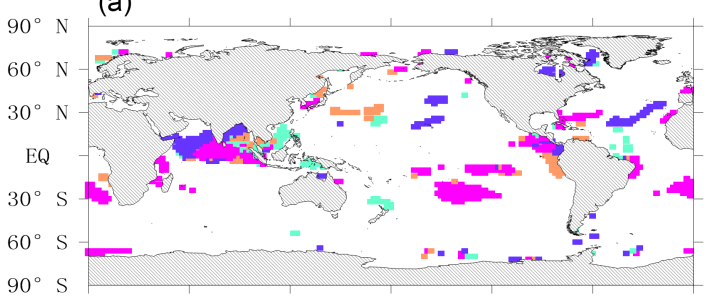

(b)

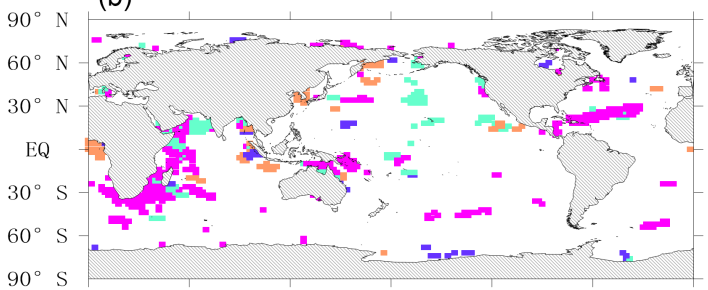

(c)

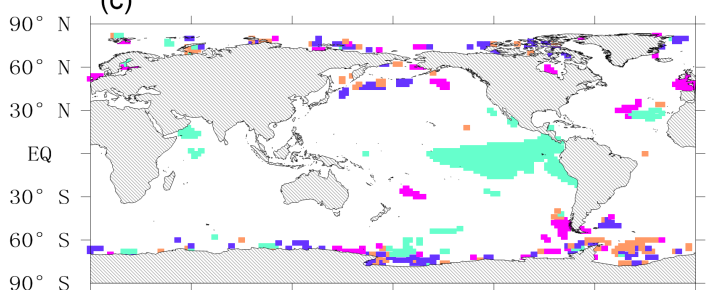

(d)

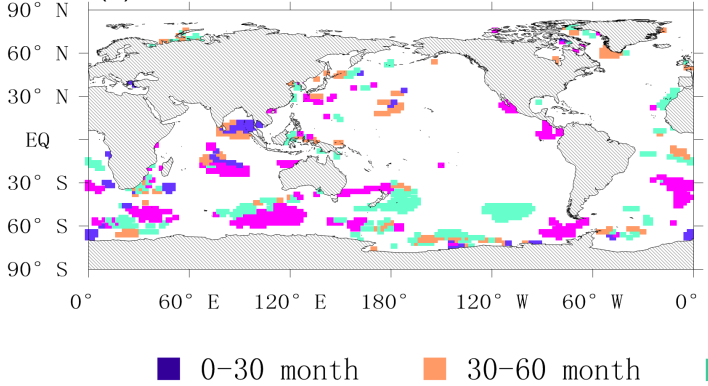

(e)

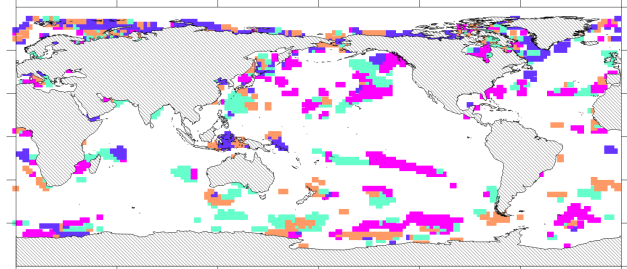

(f)
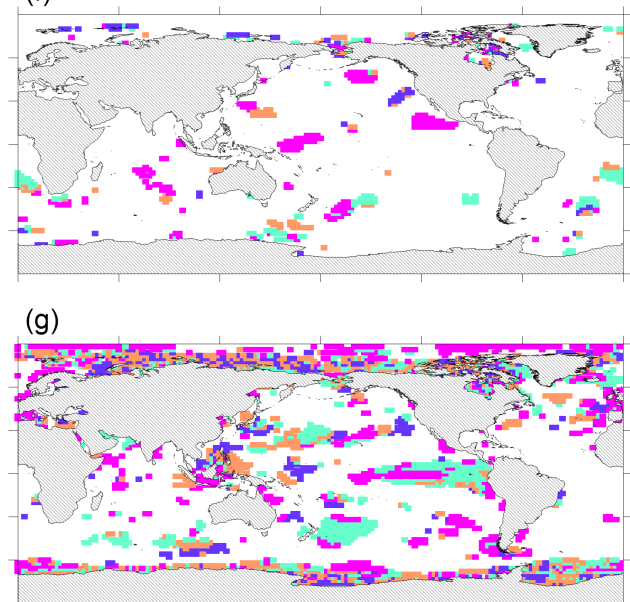

(h)

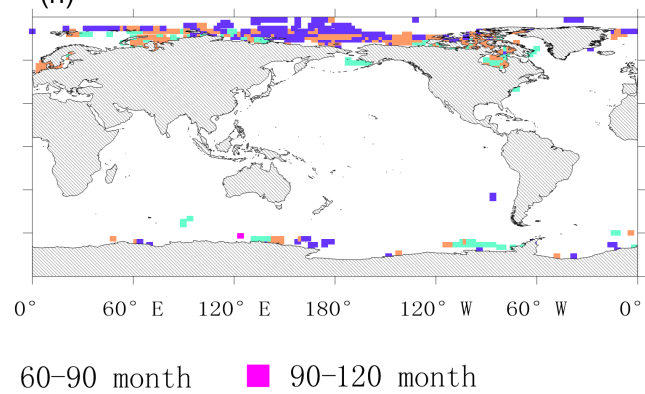

Figure 4. Spatial distribution of abrupt change duration in different periods: (a) is the abrupt change in 1878, (b) is the abrupt change in 1896-1898, (c) is the abrupt change in 1900-1903, (d) is the abrupt change in 1908-1909, (e) is the abrupt change in 1942, (f) is the abrupt change in 1976, (g) is the abrupt change in 1989-1999, and (h) is the abrupt change in 2005-2006.

\subsection{Spatial distribution of abrupt change duration}

With the start and end moments of a certain abrupt change given in the method, the period of the abrupt change may be determined. Based on this, the spatial distribution of the abrupt change duration is obtained as shown in Fig. 4: deep azure signifies that the duration is no longer than 30 months, orange signifies that the duration is about 30 60 months, turquoise signifies that the duration is about 60 90 months, and magenta signifies that the duration is about 90-120 months.

According to the start moment, eight figures of different period ACPs are analyzed:

1. For the abrupt changes starting in 1878 shown in Fig. $4 \mathrm{a}$, the duration times of the abrupt changes oc- curring in areas of the Northern Hemisphere were short, such in as the north Indian Ocean, central North Pacific, and some regions of the North Atlantic, while those of the abrupt changes occurring in the Southern Hemisphere were long, such as in the central South Pacific.

2. For the abrupt changes starting in 1896-1898 shown in Fig. 4b, mainly occurring in the south of the Southern Hemisphere, the duration times were all greater than 60 months. That in the Pacific Ocean was 60 90 months, and that in the southern Indian Ocean was slightly longer.

3. For the abrupt change starting in 1900-1903, shown in Fig. $4 c$, mainly occurring in the Pacific Ocean, the overall duration time was long (more than 60 months); and 
(a)

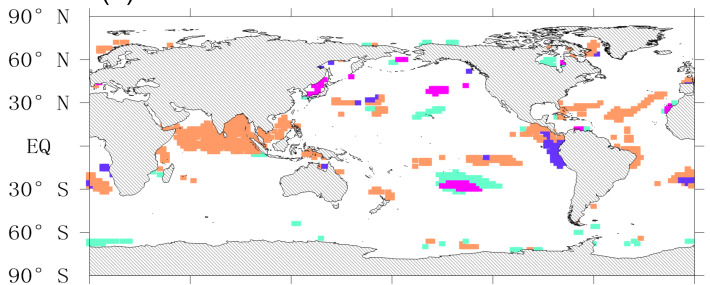

(b)

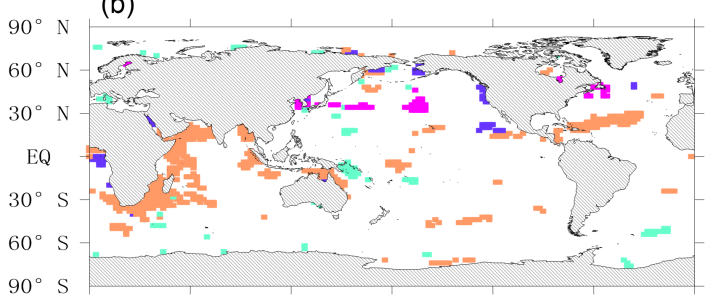

(c)

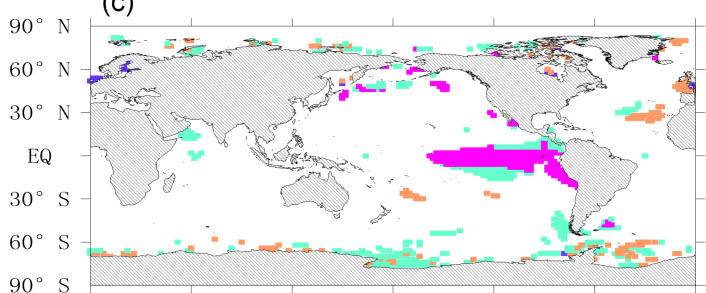

(d)

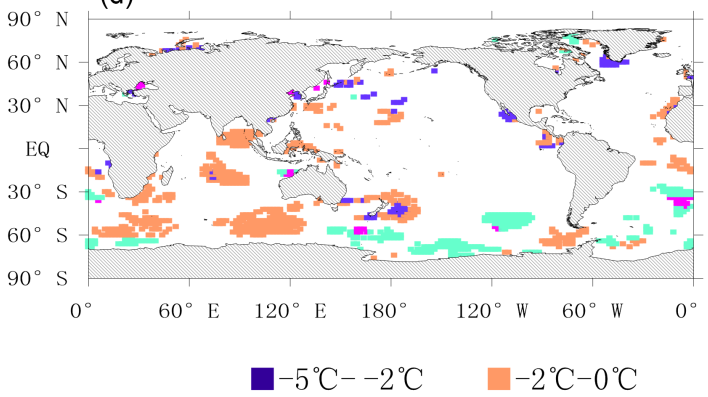

(e)
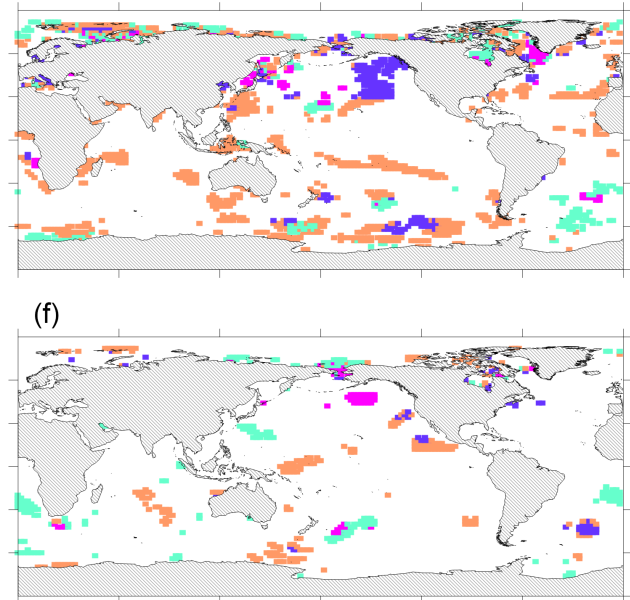

(g)

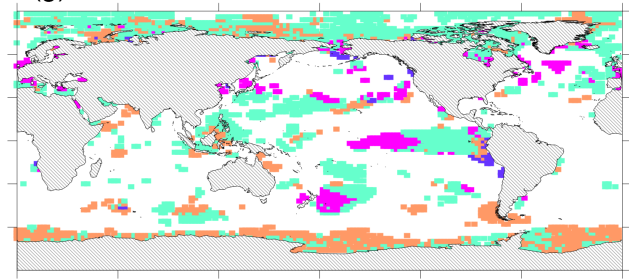

(h)

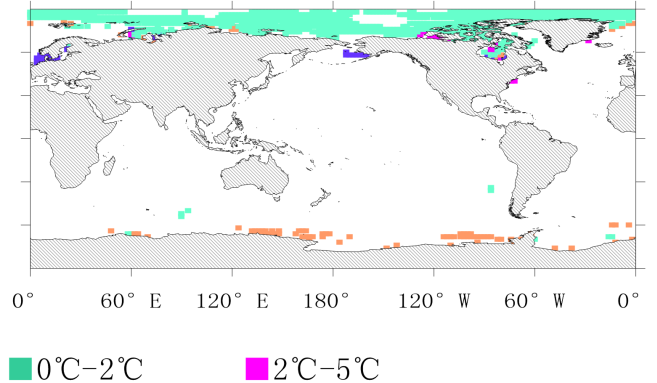

Figure 5. Amplitude distributions of abrupt changes in different periods: (a) is the abrupt change in 1878, (b) is the abrupt change in 18961898 , (c) is the abrupt change in 1900-1903, (d) is the abrupt change in 1908-1909, (e) is the abrupt change in 1942, (f) is the abrupt change in 1976, (g) is the abrupt change in 1989-1999, and (h) is the abrupt change in 2005-2006.

that in the eastern part was 90-120 months, longer than that in the western part, which was 60-90 months.

4. For abrupt change starting in 1908-1909, shown in Fig. 4d, mainly occurring in parts of the coastal regions of the Pacific and southern Indian oceans, the regions were small, while the overall duration time was long, all being longer than 60 months.

5. For the abrupt change starting in 1942, shown in Fig. 4e, the overall duration times were long throughout the world, especially in the coastal regions of the western Indian Ocean and central Atlantic, both being about 90120 months.
6. For the abrupt change starting in 1976, occurring mainly in the equatorial middle Pacific, as shown in Fig. 4f, the duration time was 60-90 months.

7. The duration time of abrupt change starting in 19891999 was relatively short, as shown in Fig. 4g. The duration time in the polar regions and western Pacific Ocean were shorter than 60 months, while that in the middleeast equatorial Pacific was a little longer, being longer than 60 months.

8. For the abrupt change starting in 2005-2006, shown in Fig. 4h, mainly occurring in the Arctic region, the duration time was less than 30 months.

Through the above analysis, the following observations are made: the duration times of abrupt change before the $1960 \mathrm{~s}$ 
(a)

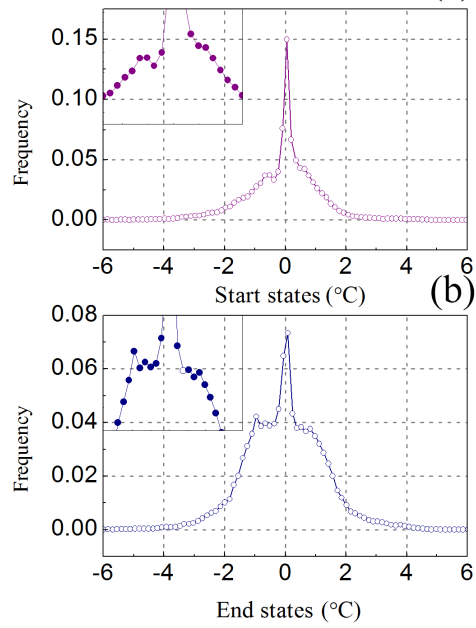

(c)

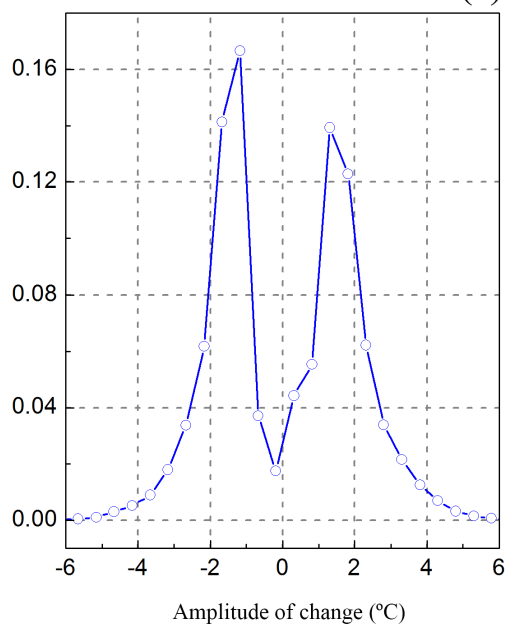

Figure 6. Bistability state characteristics study of SST system: (a) and (b) are the system state variables before and after abrupt change, respectively; and (c) is the variation quantity in the ACP of the system, namely, abrupt change amplitude.

were 60-120 months, and most were longer than 90 months; those in the 1970s were 60-90 months; those in the 1980s and 1990s were mostly 30-60 months; and those at the start of the 21 st century were shorter than 30 months. Therefore, it is understood that the duration time of abrupt change under the background of global warming has shortened.

\subsection{Spatial distribution of abrupt change amplitude parameter}

The different degree of abrupt change amplitude in different period is shown in Fig. 5: deep azure signifies that the cooling amplitude is larger than $2^{\circ}$, orange signifies that the cooling amplitude is less than $2^{\circ}$, turquoise signifies that the warming amplitude is less than $2^{\circ}$, and magenta signifies that the warming amplitude is greater than $2^{\circ}$.

According to the start moment, eight abrupt changes of different period are analyzed:

For the abrupt change starting in 1878, as shown in Fig. 5a, only in the northern region of South Pacific did the temperature increase slightly; those in the other regions (especially in Indian Ocean) decreased, and the amplitude was less than $2^{\circ}$.

The abrupt change starting in 1890-1920 is shown in Fig. 5b, c, and d; the temperature decreased in most areas, while in some it increased. The specific situations are as follows: for the abrupt change starting in 1896-1897, shown in Fig. 5b, the temperature increased by a small degree only in the south of the South Pacific; it decreased in all other regions, and the amplitude was less than $2^{\circ}$; for the abrupt change starting in 1900-1993, shown in Fig. 5c, the temperature decreased overall, and the decrease amplitude in the eastern region of the North Pacific was large (larger than $5^{\circ}$ ); for the abrupt change starting in 1908-1909, shown in
Fig. 5d, the temperatures decreased mainly in the equatorial regions, and increased in the eastern and western regions of the North Pacific and western region of the South Pacific.

For the abrupt change starting in 1942, shown in Fig. 5e, the temperatures in the coastal region of the western Indian Ocean mostly decreased (the amplitude was less than $2^{\circ}$ ), and those in some regions of the North Pacific mostly increased.

Figure $5 f$ is the abrupt change starting in 1976; the temperatures throughout the entire region increased, and the amplitude was large (larger than $2^{\circ}$ ).

Figure $5 \mathrm{~g}$ and $\mathrm{h}$ show the abrupt changes starting in 1990-2010. The temperatures increased almost everywhere throughout the world, and decreased only in some regions of the South Pole starting in 1989-1999. The temperatures in other regions all increased, and the amplitude in the central regions of equatorial Pacific was larger than $2^{\circ}$. The temperature of abrupt change starting in 2005-2006 mainly increased in the Arctic region.

Based on the above analysis, it is believed that the temperatures of abrupt changes before the 1960s mostly decreased; after experiencing the temperature rise in the 1970s, the temperatures of abrupt change throughout the world mostly increased.

\section{Statistical characteristics of parameters}

\subsection{Multistability/bistability state characteristics of abrupt climate change system}

The difference between different states of the ACP is counted in Fig. 6, and Fig. 6a shows the statistics of the start states. It is observed that the statistical probabilities of two states are high, which indicates that these states are stable. The statis- 

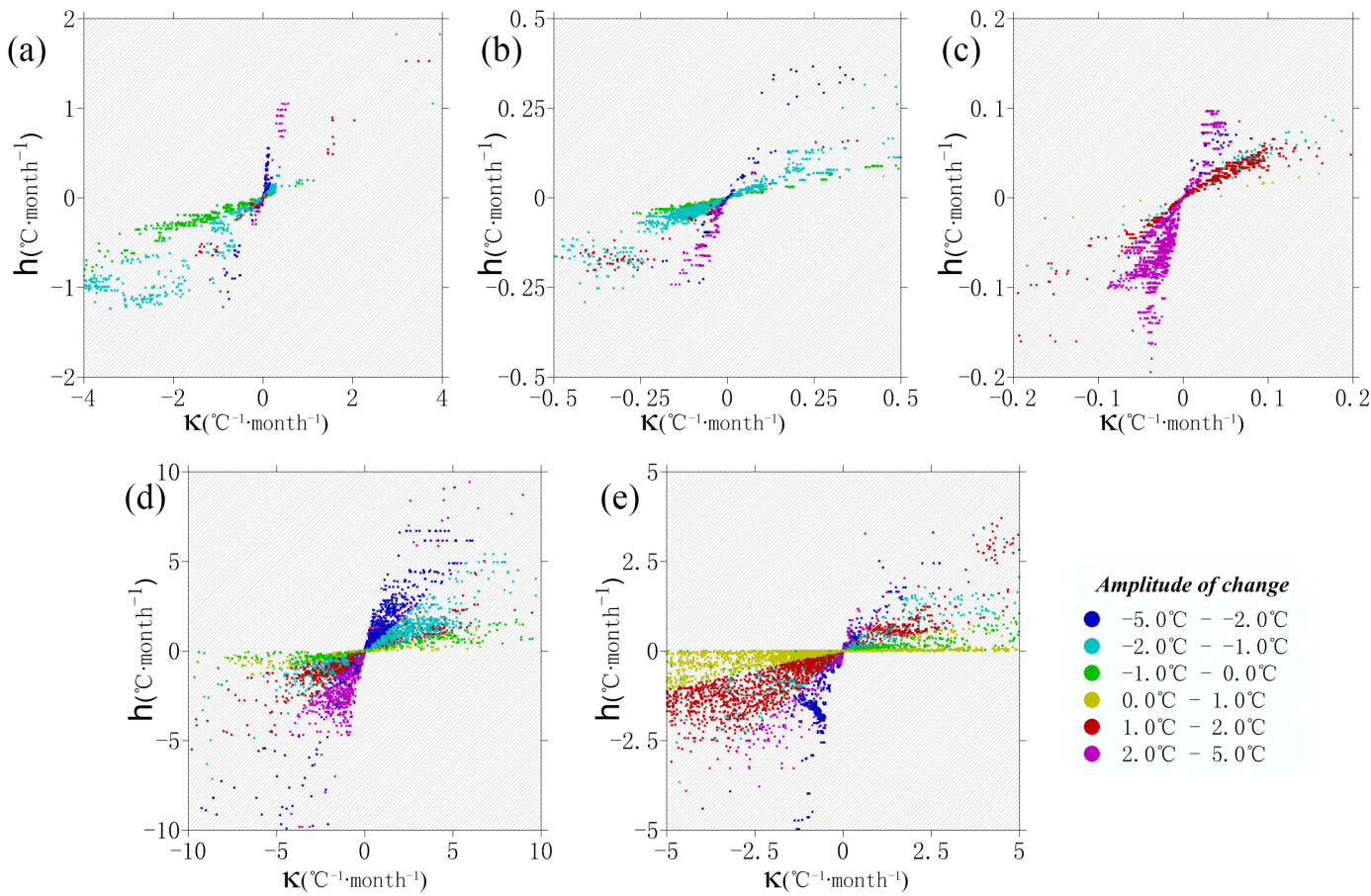

Figure 7. Relationships between abrupt change rate $h$ and instability parameter $\kappa$ in different periods and different color represent different degree by abrupt change amplitude parameter: (a-c) show the abrupt changes in 1878, 1942, and 1976, respectively; and (d-e) show the abrupt changes in 1890-1920 and 1990-2010.

tics of the end states are shown in Fig. 6b, and the statistical probabilities of the multiple states are large, which indicates that multiple stability states appear after abrupt changes of the system. After further study of the abrupt change amplitude parameter of the system, as shown in Fig. 6c, it is observed that the change amplitude parameter of the system presents a double-peak structure, and the two peaks are distributed symmetrically around the "zero point". This indicates that the system shifts in different states, and abrupt change is formed. Scholars have previously achieved similar conclusions in the study of climate system theories, i.e. the climate system is a multistability system (Baker and Charlson, 1990; Alexander et al., 2012). The results shown in Fig. 7 further prove that bistability or multistability states exist in the sea surface temperature.

\subsection{Quantitative relationship among abrupt change rate, abrupt change amplitude, and instability parameter}

The quantitative relationship among abrupt change rate $h$, instability parameter $\kappa$, and abrupt change amplitude parameter $\omega$ has previously been theoretically analyzed, and testified by the theoretical model. In this paper, by taking advantage of parameters based on the global SST sequence over the past 100 years, this conclusion is further proven using actual data. In view of the abrupt changes in 1878, 1942, 1976, in 18901920 and 1990-2010, the distribution relationships of param- eters $h-\omega$ and $h-\kappa$ of each period sequence are respectively shown in Figs. 7 and 8 successively.

Figure 7 shows the relationship between abrupt change rate $h$ and instability $\kappa$. Figure 7a-e are the abrupt changes in different periods. Taking the abrupt change of 1942 in Fig. $7 \mathrm{~b}$ as an example, the horizontal axis is the instability $\kappa$, and the vertical axis is the abrupt change rate $h$. With the increase of instability parameter, the abrupt change rate increases with a direct proportion, showing a direct proportion between the two. In terms of the numerical value of successive abrupt change, the instabilities of abrupt change in 1878 , 1942 , and 1976 are within the range of -0.15 to +0.15 . The three abrupt changes all occurred in regions of middle and low latitude. However, for the abrupt changes in 1890-1920 and 1990-2010 occurring in regions of high latitude, the instabilities are within the range from -4 to +4 . This indicates that the system stability of high latitude areas is lower than that of low latitude areas.

Figure 8 shows the relationship between abrupt change rate $h$ and abrupt change amplitude parameter $\omega$. Taking the abrupt changes in 1990-2010 in Fig. 8e as an example, the horizontal axis is the abrupt change amplitude parameter, and the vertical axis is the abrupt change rate. The rate increases with the increase of change amplitude parameter, and presents a parabolic increase. This indicates that the increase of abrupt change rate is doubled with the increase of change amplitude parameter. This also explains the phe- 

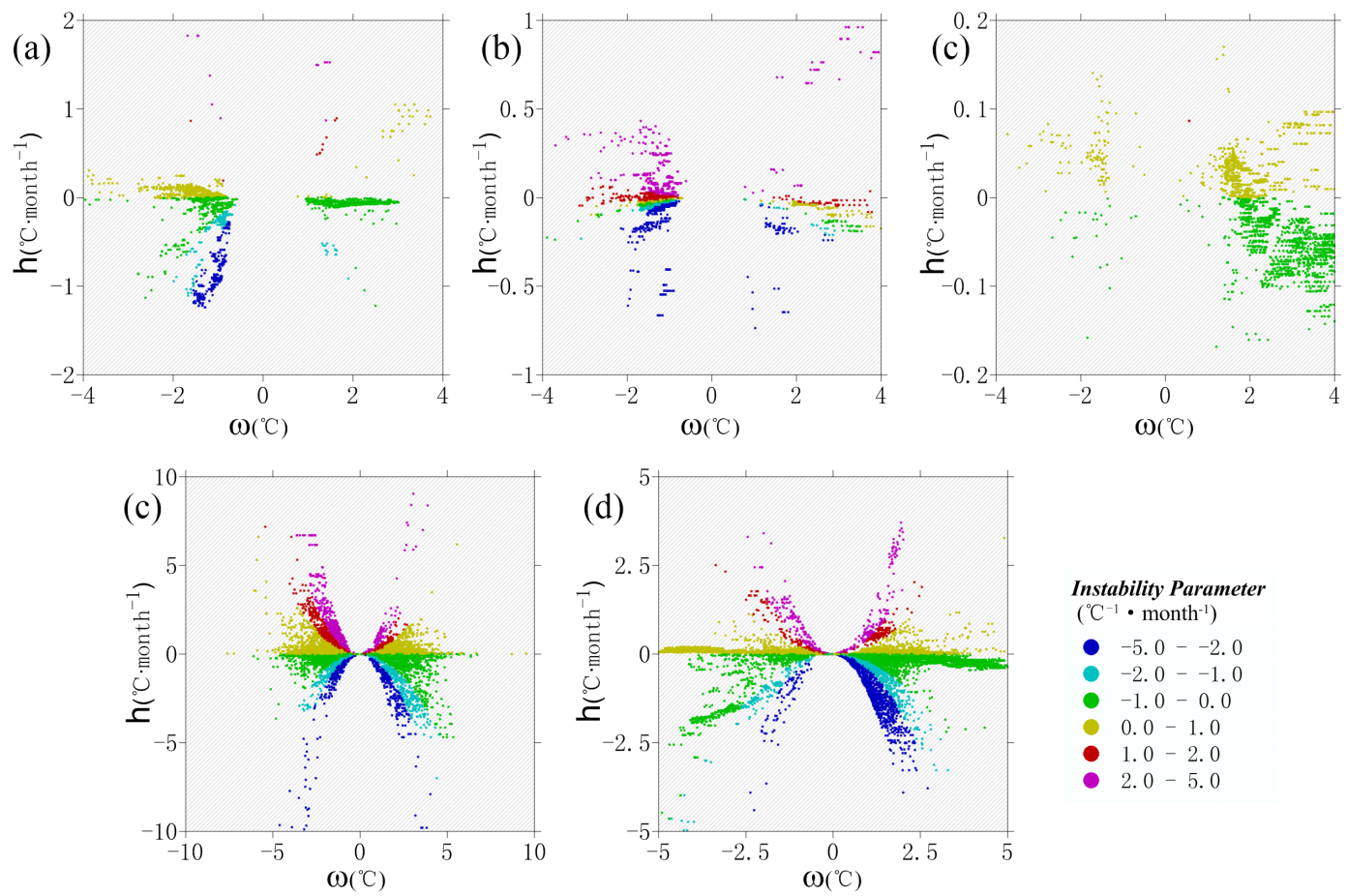

Figure 8. Relationship between abrupt change rate $h$ and abrupt change amplitude parameter $\omega$, and different colors represent different degrees by instability amplitude: (a-c) show the abrupt changes in 1878, 1942, and 1976; and (d-e) are the abrupt changes in 1890-1920, 1990-2010.

nomenon whereby the faster the speed of the abrupt change is, the larger the change amplitude parameter, causing immense destructive effects on the environment.

In the results above, the Figs. $7-8$ prove the quantitative relationship provided in Eq. (8), i.e., the abrupt change rate and instability are in direct proportion, and the abrupt change rate is in direct proportion with the quadratic abrupt change amplitude parameter. Based on this relationship, the existing abrupt change amplitude parameter of the actual climate sequence can be used to estimate the abrupt change rate and further estimate the stability of the system. It is also noticed that instability is a parameter related to the property of the system itself, and with a large amount of observed statistical data the range may be obtained. This may then be used in the estimation of abrupt change of the system, and for the further estimation of future abrupt change amplitude parameters.

\section{Conclusions and expectations}

The climate is a gigantic, complex, and chaotic system with bistable (multistable) states. Its transition from one state to another is considered to be an abrupt climate change. In the present paper, by applying a novel transition process detection method, the SST system is verified to be bistable and several abrupt changes are detected. The results also provide an understanding of the transition period, in which the system has already left its original state, but has still not reached its new state:
1. The transition process is a significant period of an abrupt change event. According to the start and end moment, the abrupt change event can be divided into three stages. Based on GSST series, several abrupt changes have been identified in the past 100 years, and the spatial distribution reveals that abrupt change amplitudes in low-latitude regions are small, but those in regions of middle and high latitude are large.

2. In view of these characteristics of the abrupt change process, the spatial distribution of abrupt change duration is analyzed. It is discovered that abrupt changes in different periods have different durations. The durations of abrupt changes before 1942 were generally long (about 5-10 years); that in 1976 was about 5-8 years; and those after this time were short (about 5 years). This indicates that the durations being shortened by abrupt change are consistent with global warming.

3. The spatial distribution of abrupt change amplitudes indicates that the temperature decreased throughout the world mostly before 1942, but mostly increased after that. The statistical results for abrupt change amplitude verifies that the SST is bistable and that the system state variables have shifted many times from one stable state to another. 
4. By analyzing the parameters estimated from the SST system, a quantitative relationship is demonstrated that the relationship between the rate of abrupt change and stability is linear, and the relationship between the rate of abrupt change and change amplitude is quadratic. This observation reveals the cause of the high rate and large amplitude of abrupt climate change.

In the present paper, the transition process of abrupt climate change is detected, based on reanalysis data from (NOAA) National Oceanic and Atmospheric Administration, and a quantitative relationship is exposed. With the quantitative relationship, further research about the prediction of abrupt climate change is worthy of further examination.

\section{Data availability}

The authors acknowledge the National Oceanic and Atmospheric Administration (Earth System Research Laboratory, 2016) for providing extended reconstructed sea surface temperature (SST) V4 (http://www.esrl.noaa.gov/psd/ data/gridded/data.noaa.ersst.html).

Acknowledgements. This study was jointly sponsored by the National Natural Science Foundation of China (grants 41305056, 41530531, 41375069), the key Special Scientific Research Fund of Meteorological Public Welfare Profession of China (grant GYHY201506001), and the State Key Development Program for Basic Research of China (grant 2013CB430204).

Edited by: V. Perez-Munuzuri

Reviewed by: two anonymous referees

\section{References}

Alexander, R., Reinhard, C., and Andrey, G.: Multistability and critical thresholds of the Greenland ice sheet, Nature Climate Change, 2, 429-432, 2012.

Baker, M. B. and Charlson, R. J.: Bistability of CCN concentrations and thermodynamics in the cloud-topped boundary layer, Nature, $345,142-145,1990$.

Charney, J. G. and DeVore, J. G.: Multiple flow equilibria in the atmosphere and blocking, J. Atmos. Sci., 36, 1205-1216, 1979.

Earth System Research Laboratory: NOAA_ERSST_V4, available at: http://www.esrl.noaa.gov/psd/data/gridded/data.noaa. ersst.html, last access: 17 May 2005.

Feng, G. L., Hou, W., Zhi, R., Yang, P., Zhang, D. Q., Gong, Z. Q., and Wan, S. Q.: Research on Detecting, Diagnosing and Predictability of Extreme Climate Events, Science Press, Beijing, China, 2011.

$\mathrm{Fu}, \mathrm{C}$. B. and Wang, Q.: The definition and detection of the abrupt climate changr, Scientia atmospherica sinica, 16, 482-493, 1992.

Goldblatt, C., Lenton, T. M., and Watson, A. J.: Bistability of atmospheric oxygen and the Great Oxidation, Nature, 443, 683-686, 2006.
Guttal, V. and Jayaprakash, C.: Changing skewness, an early warning signal of regime shifts in ecosystems, Ecol. Lett., 11, 450460, 2008.

He, W. P., Wang, Q. G., Wu, Q., Zhang, W., and Zhang, Y.: Comparison of characteristics of moving detrended fluctuation analysis with that of approximate entropy method in detecting abrupt dynamic change, Acta Phys. Sin., 58, 2862-2871, 2009.

He, W. P., Feng, G. L., Wu, Q., He, T., Wan, S. Q., and Chou, J. F.: A new method for abrupt dynamic change detection of correlated time series, Int. J. Climatol., 32, 1604-1614, 2012.

Hirahara, S., Ishii, M., and Fukuda, Y.: Centennial-scale sea surface temperature analysis and its uncertainty, J. Climate, 27, 57-75, 2014.

IPCC: Climate Change 2014, Synthesis Report, Cambridge University Press, Cambridge, UK, 2014.

Jin, H. M., He, W. P., Liu, Q. Q., Wang, J. S., and Feng, G. L.: The applicability of research on moving cut data-approximate entropy on abrupt climate change detection, Theor. Appl. Climatol., 124, 475-486, 2015.

Kendall, M. G: Rank Correlation Methods, Griffin, London, UK, 1955.

Kendall, M. G. and Stuart, A.: The Advanced Theory of Statistics, Vol. I: Distribution Theory, Griffin, London, UK, 1976.

Li, J. P., Chou, J. F., and Shi, J. E.: Complete definition and types of abrupt climate change, Journal of Beijing Meteorological college, 1, 7-12, 1996.

Liu, B. Z. and Peng J. H.: Nonlinear Dynamics, Higher Education Press, Beijing, China, 215-218, 2004.

Liu, W., Huang, B., Thorne, P. W., Banzon, V. F., Zhang, H., Freeman, E., Lawrimore, J., Peterson, T. C., Smith, T. M., and Woodruff, S. D.: Extended Reconstructed Sea Surface Temperature Version 4 (ERSST.v4), Part II, Parametric and Structural Uncertainty Estimations, J. Climate, 28, 931-951, 2015.

Lorenz, E. N.: Deterministic Non-periodic Flow, J. Atmos. Sci., 20, 130-141, 1963.

Lorenz, E. N.: Nondeterministic theories of Climate change, Quaternary Res., 6, 495-506, 1976.

Mann, H. B.: Nonparametric tests against trend, Econometrica, 13, 245-259, 1945

May, R.: Simple mathematical models with very complicated dynamics, Nature, 261, 459-467, 1976.

Mudelsee, M.: Ramp function regression: a tool for quantifying climate Transitions, Comput. Geosci., 26, 293-307, 2000

Ratna, S. B., Cherchi, A., Joseph, P. V., Behera, S. K., Abish, B., and Masina, S.: Moisture variability over the Indo-Pacific region and its influence on the Indian summer monsoon rainfall, Clim. Dynam., 46, 949-965, 2015

Rial, J. A.: Abrupt climate change, chaos and order at orbital and millennial scales, Global Planent. Change, 41, 95-105, 2004.

Suryachandra, A. R., Swadhin, K. B., Yukio, M., and Toshio, Y.: Interannual subsurface variability in the Tropical Indian Ocean with a special emphasis on the Indian Ocean Dipole, Deep-Sea Res. Pt. II, 49, 1549-1572, 2002.

Thom, R.: Stability Structural and Morphogenesis, Sichuan Education Press, Sichuan, China, 1972.

Wei, F. Y.: Modern Climatic Statistical Diagnosis and Forecasting Technology, China Meteorological Press, Beijing, China, 1999.

Xiao, D. and Li, J. P.: Spatial and temporal characteristics of the decadal abrupt changes of global atmosphere- 
ocean system in the 1970s, J. Geophys. Res., 112, D24S22, doi:10.1029/2007JD008956, 2007.

Yamamoto, R., Iwashima, T., and Sanga, N. K.: An analysis of climatic jump, J. Meteorol. Soc. JPN, 64, 273-281, 1986.

Yan, G. H., Yan, P. C., Hou, W., and Wu, H.: A method of abrupt change process analysis based on Logistic model and its applications, Acta Phys. Sin., 62, 079202, doi:10.7498/aps.62.079202, 2013.

Yan, P. C., Hou, W., and Hu, J. G.: The critical warning research of the mean time series mutations based on Logistic model, Acta Phys. Sin., 61, 189202, doi:10.7498/aps.61.189202, 2012.
Yan, P. C., Feng, G. L., Hou, W., and Wu, H.: Statistical characteristics on decadal abrupt change process of time sequence in $500 \mathrm{hPa}$ temperature field, Chinese Journal of Atmospheric Sciences, 38, 861-873, 2014.

Yan, P. C., Feng, G. L., and Hou, W.: A novel method for analyzing the process of abrupt climate change, Nonlin. Processes Geophys., 22, 249-258, doi:10.5194/npg-22-249-2015, 2015.

Zerkle, A. L., Claire, M. W., Domagal-Goldman, S. D., Farquhar, J., and Poulton, S. W.: A bistable organic-rich atmosphere on the Neoarchaean Earth, Nat. Geosci., 5, 359-363, 2012. 